\title{
Editorial
}

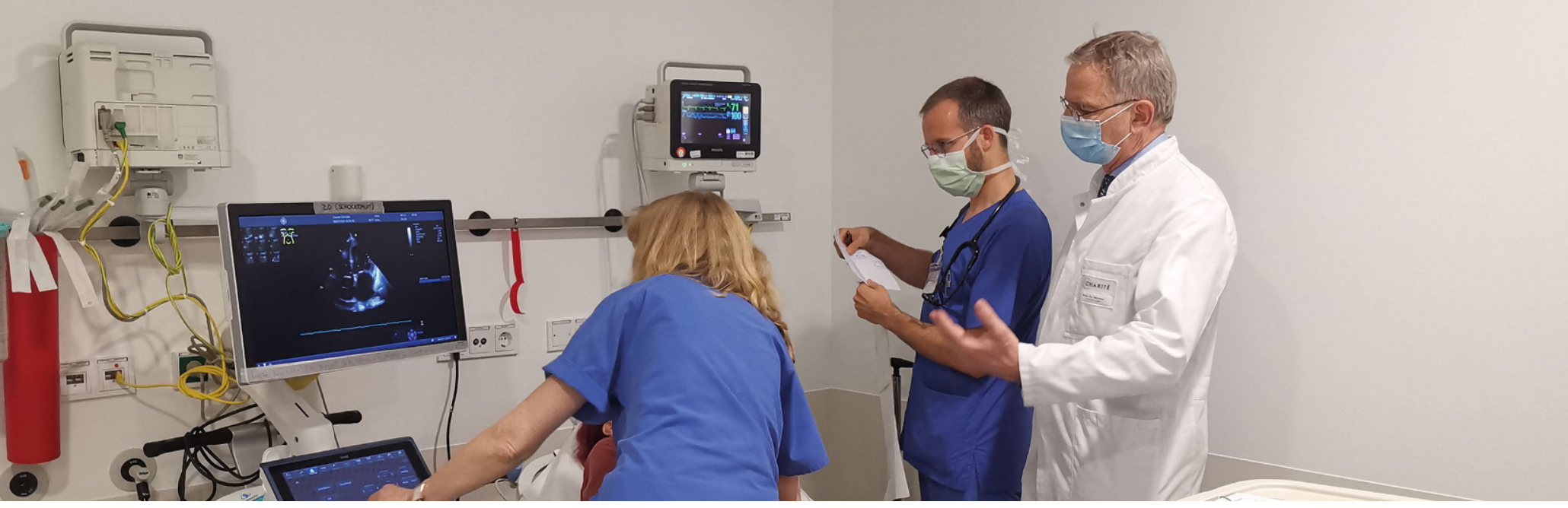

Internist 2021 - 62 (Suppl 4):S353-S355 https://doi.org/10.1007/s00108-021-01137-3 Angenommen: 29. Juli 2021

Online publiziert: 3. September 2021

(c) Springer Medizin Verlag $\mathrm{GmbH}$, ein Teil von Springer Nature 2021

Q)

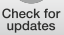

\section{Facharzt-Training Innere Medizin - Das dritte Sonderheft ist da!}

\section{Fallbezogenes Lernen anhand der neuen Muster- Weiterbildungsordnung}

\author{
Georg Ertl ${ }^{1}$ Alexander Arlt ${ }^{2}$ \\ ' Deutsches Zentrum für Herzinsuffizienz, Universitätsklinikum Würzburg, Würzburg, Deutschland \\ ${ }^{2}$ Universitätsklinik für Innere Medizin - Gastroenterologie, Klinikum Oldenburg AöR, Oldenburg, \\ Deutschland
}

Der Facharzt für Innere Medizin ist zu Zeiten der voranschreitenden Spezialisierung in den Teilgebieten der Inneren Medizin und darüber hinaus notwendiger denn je. Die Betreuung der älteren und häufig multimorbiden Patient*innen erfordert neben der Fachexpertise für das jeweilige Teilgebiet ein breites Wissen in der Inneren Medizin.

\section{Kompendium für die Facharzt- prüfung}

Das dritte Sonderheft Facharzt-Training Innere Medizin setzt das geschätzte Konzept eines fallbasierten Kompendiums fort. Das Wissen zu den wichtigen Erkrankungen aus dem internistischen Alltag wird in hoher Qualität von Fachleuten aufbereitet. Anhand typischer und untypischer Fälle wird die aktuelle State-of-the-Art-Diagnostik und -Therapie praxisnah vermit- telt - für die Prüfung, aber auch für die Praxis. Das Facharzt-Training Innere Medizin eignet sich ideal für die Vorbereitung auf die Facharztprüfung und bietet - auch zum Nachschlagen - einen guten Überblick über Erkrankungen, Diagnostik und Therapie.

\section{Halbzeit mit 75 Prüfungsfällen}

Die Beiträge des dritten Sonderhefts zu Themen wie chronisch-obstruktive Lungenerkrankung, Diabetes, Sepsis, akutes Koronarsyndrom und Reflux sind konsequent praxisorientiert aus allen Bereichen der Inneren Medizin. Auch in dieser Ausgabe haben wir uns in Zusammenschau mit den ersten beiden Heften um eine breite Abbildung aller Teilgebiete bemüht. In den nächsten Monaten wird die Serie mit weiteren Sonderheften fortgesetzt, bis die 


\begin{tabular}{|l|l|}
\hline $\begin{array}{l}\text { Tab. 1 Verteilung der Prüfungsfallthe- } \\
\text { men auf die Fachgebiete }\end{array}$ \\
\hline $\begin{array}{l}\text { Facharzt-Training Innere Medizin } \\
\text { Stand September } 2021\end{array}$ \\
\hline Angiologie & 7 \\
\hline Diabetologie und Endokrinologie & 11 \\
\hline Gastroenterologie & 12 \\
\hline Geriatrie & 3 \\
\hline Hämatologie und Onkologie & 6 \\
\hline Infektiologie & 12 \\
\hline Kardiologie & 8 \\
\hline Nephrologie & 4 \\
\hline Pneumologie & 8 \\
\hline Rheumatologie & 4 \\
\hline Summe & 75 \\
\hline
\end{tabular}

geplanten 150 Prüfungsfallthemen vorliegen (• Tab. 1).

\section{Facharzt-Training online und in der App}

Begleitend zu den Sonderheften gibt es die Inhalte online als E-Learning über die DGIM e.Akademie und auch in der Springer Medizin App "Facharzt Training“ (erhältlich über die bekannten App-Stores). So kann jeder für sich entscheiden, ob er sich per Sonderheft oder lieber digital mit mittlerweile 75 Prüfungsfällen auf die Facharztprüfung vorbereiten möchte.

\section{Fortbildungsprogramm DGIM e.Akademie}

Wir möchten auf das E-Learning-Angebot der Deutschen Gesellschaft für Innere Medizin (DGIM) und des Springer Medizin Verlags hinweisen. Jedes Mitglied der DGIM hat Zugriff auf das Fortbildungsangebot der DGIM e.Akademie. Hier finden Sie das Facharzt-Training und auch das umfangreiche CME-Fortbildungsangebot zu verschiedenen Schwerpunkten und Zusatzgebieten der Inneren Medizin. Über einen Newsletter werden Sie regelmäßig informiert. Im Internet finden Sie das Programm unter: https://www.dgim-eakademie.de.

Das Facharzt-Training Innere Medizin wird vom Wissenschaftlichen Beirat der DGIM im Rahmen der DGIM e.Akademie herausgegeben (- Infobox 1).

\section{Infobox 1}

Herausgeberschaft und Beirat Facharzt-

Training Innere Medizin

Herausgeber

- Prof. Dr. med. Alexander Arlt, Oldenburg (fdf. Herausgeber)

- Dr. med. Mariam Abu-Tair, Bielefeld

- Dr. med. Birgit Bader, Berlin

- Prof. Dr. med. Georg Ertl, Würzburg

- Prof. Dr. med. Dr. h.c. Ulrich R. Fölsch (Ehrenherausgeber)

- Dr. med. Manuela Hoechstetter, München

- Prof. Dr. med. Martin Möckel, Berlin

- Prof. Dr. med. Oliver Müller, Kiel

- Dr. med. Matthias Raspe, Berlin

- Prof. Dr. med. Sebastian Schmid, Lübeck

- PD Dr. med. Philipp Sewerin, Düsseldorf

- PD Dr. med. Ulrich Seybold, München

- Prof. Dr. med. Katrin Singler, Nürnberg

Beirat

- Dr. med. Antje Auler, Berlin

- Dr. med. Ulrike Bachmann, Berlin

- Dr. med. Anahita Fathi, Hamburg

- Dr. med. Kai Hasenclever, Köln

- PD Dr. med. Dr. med. nat. Urs Lichtenauer, Schwerin

- Dr. med. Friederike Lutz, München

- Andrea Martini, Friedrichshafen

- Clara Matthiessen, Berlin

- Dr. med. Carola Misgeld, Berlin

- Sarmina Latif, Berlin

- Dr. med. Timur Özkan, Berlin

- Dr. med. Anna Schappert, Berlin

- Dr.med. Nicola Schönewolf, MSc, München

- Dr. med. Sophie Teege, Hamburg

- Dr. med. Anja Vogelgesang, Göttingen

Wir wünschen Ihnen eine spannende und bereichernde Lektüre mit diesem Sonderheft Facharzt-Training Innere Medizin.

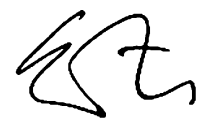

Prof. Dr. Georg Ertl

Generalsekretär der DGIM

Vorsitzender des Wissenschaftlichen Beirats der DGIM

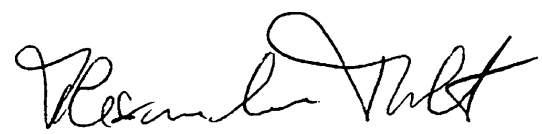

Prof. Dr. Alexander Arlt

Federführender Herausgeber FacharztTraining Innere Medizin

\section{Korrespondenzadresse}

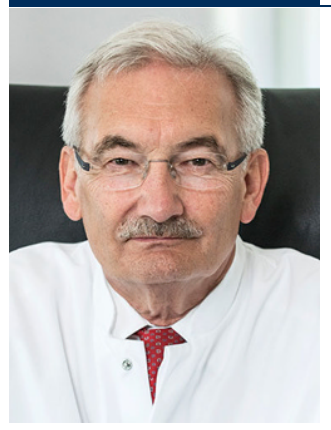

Prof. Dr. med. Georg Ertl

Oberdürrbacher Str. 6, 97080 Würzburg, Deutschland

ertl_g@ukw.de

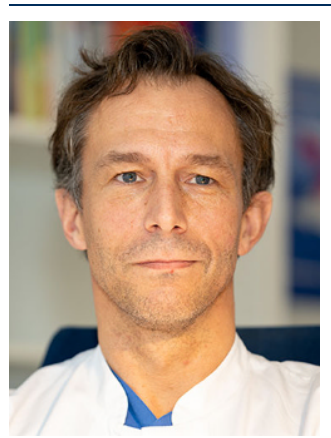

Univ.-Prof. Dr. med. Alexander Arlt

Rahel-Straus-Straße 10, 26133 Oldenburg, Deutschland

alexander.arlt@uni-oldenburg.de

Interessenkonflikt. G. Ertl und A. Arlt geben an, dass kein Interessenkonflikt besteht. 
Hier steht eine Anzeige.

黑 Springer 